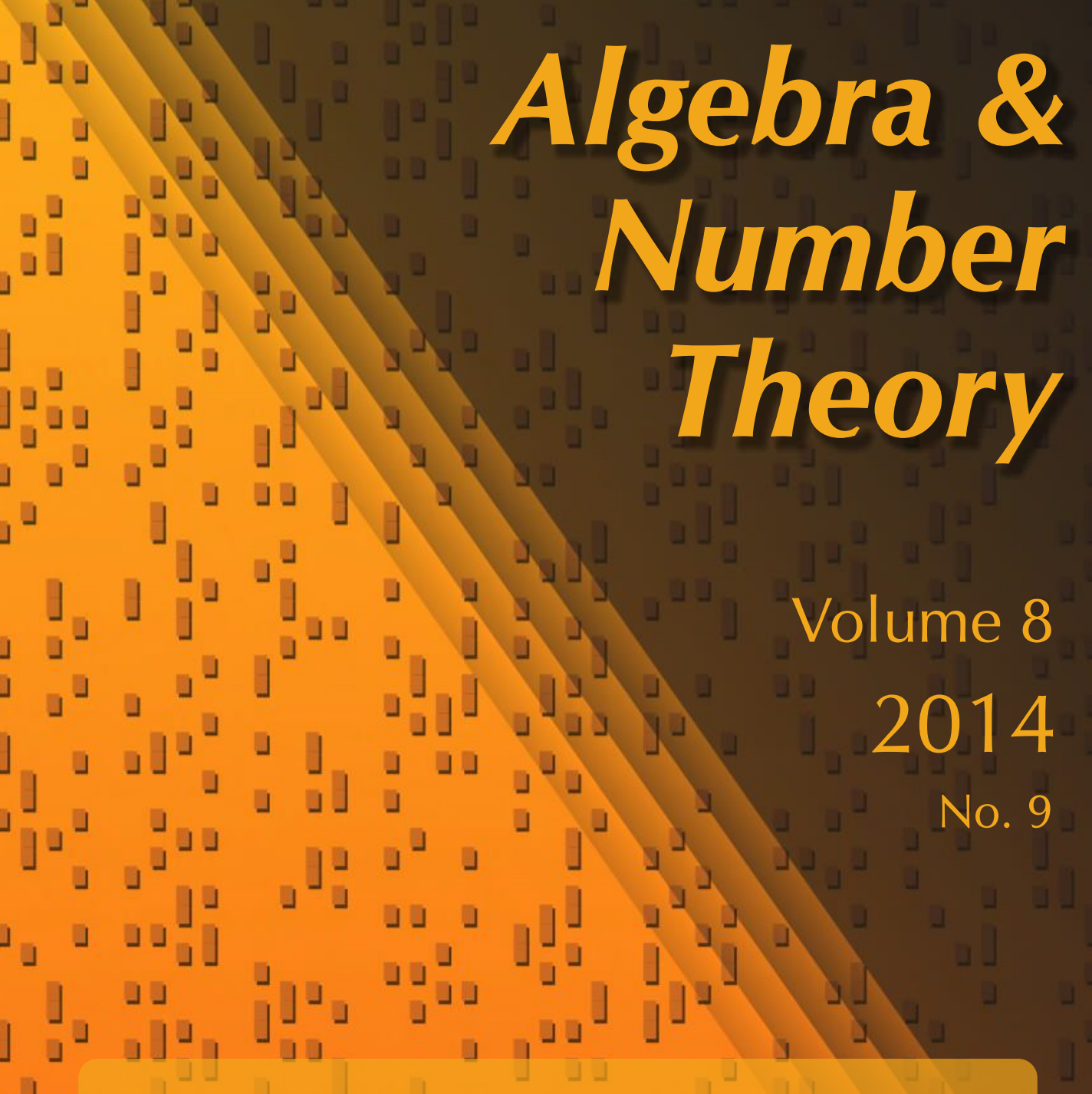

Finiteness of unramified deformation rings

Patrick B. Allen and Frank Calegari

」

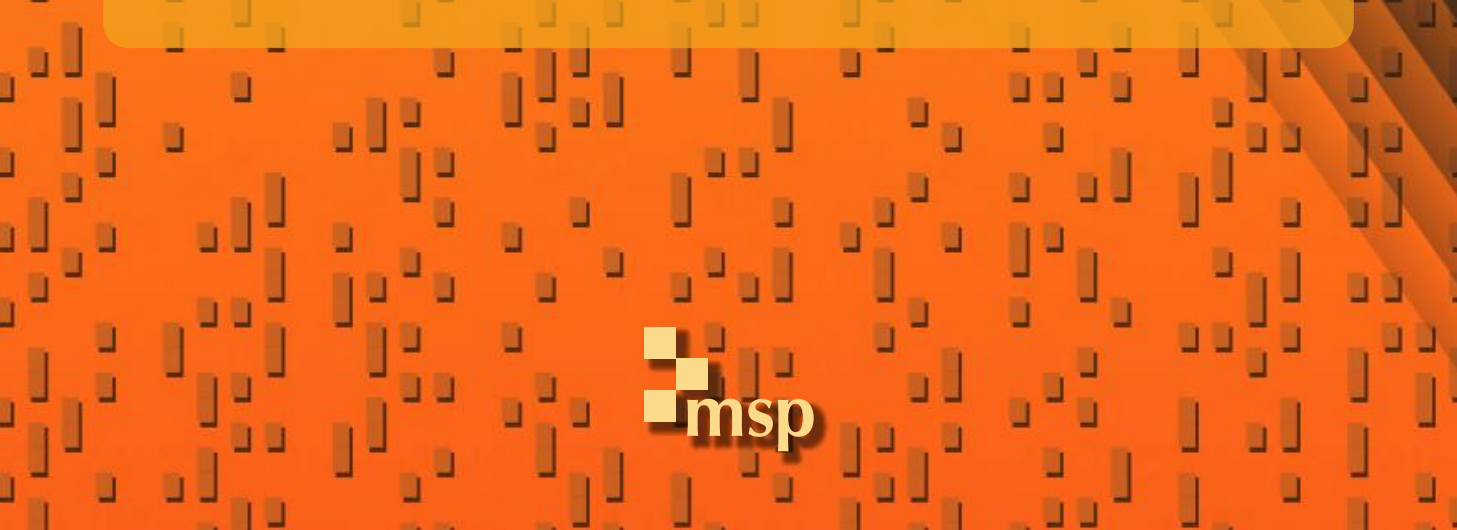




\title{
Finiteness of unramified deformation rings
}

\author{
Patrick B. Allen and Frank Calegari
}

\begin{abstract}
We prove that the universal unramified deformation ring $R^{\text {unr }}$ of a continuous Galois representation $\bar{\rho}: G_{F^{+}} \rightarrow \mathrm{GL}_{n}(k)$ (for a totally real field $F^{+}$and finite field $k$ ) is finite over $0=W(k)$ in many cases. We also prove (under similar hypotheses) that the universal deformation ring $R^{\text {univ }}$ is finite over the local deformation ring $R^{\text {loc }}$.
\end{abstract}

\section{Introduction}

Let $k$ be a finite field of characteristic $p$, and let $0=W(k)$. Let $F$ be a number field, and consider a continuous absolutely irreducible Galois representation

$$
\bar{\rho}: G_{F} \rightarrow \mathrm{GL}_{n}(k),
$$

where $G_{F}=\operatorname{Gal}(\bar{F} / F)$ for some fixed algebraic closure $\bar{F}$ of $F$. If $(A, \mathfrak{m})$ is a complete local $\mathcal{O}$-algebra with residue field $k$, then a deformation $\rho$ of $\bar{\rho}$ to $A$ unramified outside a finite set of primes $S$ consists an equivalence class of homomorphisms

$$
\rho: G_{F} \rightarrow \mathrm{GL}_{n}(A)
$$

such that the composite of $\rho$ with the projection $\mathrm{GL}_{n}(A) \rightarrow \mathrm{GL}_{n}(A / \mathfrak{m})=\mathrm{GL}_{n}(k)$ is $\bar{\rho}$, and such that the extension of fields $F(\operatorname{ker}(\rho))$ over $F(\operatorname{ker}(\bar{\rho}))$ is unramified away from places above primes in $S$ (see [Mazur 1997]). The nature of such deformations is quite different depending on whether $S$ contains the primes above $p$ or not. If $S$ contains all the primes above $p$, we denote the universal deformation ring by $R^{\text {univ }}$; if $S$ contains no primes above $p$, we denote the corresponding universal deformation ring by $R^{\mathrm{unr}}$. According to the Fontaine-Mazur conjecture (see [Fontaine and Mazur 1995, Conjecture 5a]), any map $R^{\mathrm{unr}} \rightarrow \overline{\mathbb{Q}}_{p}$ gives rise to a deformation $\rho$ of $\bar{\rho}$ with finite image. (This form of the conjecture is known as the unramified Fontaine-Mazur conjecture.) Boston's strengthening of this conjecture

Allen was supported in part by a Simons Research Travel Grant. Calegari was supported in part by NSF Career Grant DMS-0846285.

MSC2010: primary 11F80; secondary 11F70.

Keywords: Galois representations. 
[Boston 1999, Conjecture 2 and the subsequent corollary] is the claim that the universal unramified deformation

$$
\rho^{\mathrm{unr}}: G_{F} \rightarrow \mathrm{GL}_{n}\left(R^{\mathrm{unr}}\right)
$$

has finite image. In contrast, the ring $R^{\text {univ }}$ is typically of large dimension (see $\S 1.10$ of [Mazur 1989]). A conjecture of Mazur predicts that the relative dimension of $R^{\text {univ }}$ over $O$ is (in odd characteristic)

$$
\left(1+r_{2}\right)+\left(n^{2}-1\right)[F: \mathbb{Q}]-\sum_{v \mid \infty} \operatorname{dim} H^{0}\left(D_{v}, \operatorname{ad}^{0}(\bar{\rho})\right),
$$

where $\operatorname{ad}^{0}(\bar{\rho})$ denotes (in any choice of basis) the trace zero matrices in $\operatorname{Hom}(\bar{\rho}, \bar{\rho})$. A choice of basis for the universal deformation makes $R^{\text {univ }}$ an algebra over a local deformation ring

$$
R^{\mathrm{loc}}=\widehat{\bigotimes}_{v \mid p} R_{v}^{\mathrm{loc}}
$$

where $R_{v}^{\text {loc }}$ is the universal framed local deformation ring of $\bar{\rho} \mid D_{v}$ for $v \mid p$. The $R^{\text {loc }}$-algebra structure may depend on the choice of basis, but it is canonical up to automorphisms of $R^{\text {loc }}$. It is not true in general that $\operatorname{Spec}\left(R^{\text {univ }}\right) \rightarrow \operatorname{Spec}\left(R^{\text {loc }}\right)$ is a closed immersion, even in the minimal case where $S$ is only divisible by the primes dividing $p$. A simple example to consider is the deformation ring of any one-dimensional representation $\bar{\rho}: G_{F} \rightarrow k^{\times}$; the corresponding map $\operatorname{Spec}\left(R^{\text {univ }}\right) \rightarrow \operatorname{Spec}\left(R^{\text {loc }}\right)$ is a closed immersion if and only if the maximal everywhere-unramified abelian $p$-extension of $F$ in which $p$ splits completely is trivial. It is, however, reasonable to conjecture that this map is always a finite morphism. Indeed, one heuristic justification for the Fontaine-Mazur conjecture is to imagine that the generic fibers of the image of $\operatorname{Spec}\left(R^{\text {univ }}\right)$ and the locus of local crystalline representations of a fixed weight are transverse, and to infer (from a conjectural computation of dimensions) that the intersection is finite, and hence that there are only finitely many global crystalline representations of a fixed weight (see pp. 191-192 of [Fontaine and Mazur 1995]); this line of thinking at least presumes that the global-to-local map is quasifinite.

We prove the following:

Theorem 1. Let $F^{+}$be a totally real field, and let $\bar{\rho}: G_{F^{+}} \rightarrow \mathrm{GL}_{n}(k)$ be a continuous absolutely irreducible representation. Suppose that:

(1) $p>2$.

(2) $\operatorname{ad}^{0}\left(\bar{\rho} \mid G_{F^{+}\left(\zeta_{p}\right)}\right)$ is absolutely irreducible and $p>2 n^{2}-1$, or, if $n=2$ and $\bar{\rho}$ is totally odd, $\bar{\rho} \mid G_{F^{+}\left(\zeta_{p}\right)}$ has adequate image.

Then $R^{\mathrm{unr}}$ is a finite $\mathrm{O}$-algebra, and $R^{\mathrm{univ}}$ is a finite $R^{\text {loc }}$-algebra. 
The second condition holds, for example, when $\bar{\rho}$ has image containing $\mathrm{SL}_{n}(k)$ and $p$ is greater than $2 n^{2}-1$. The finiteness of $R^{\text {univ }}$ over $R^{\text {loc }}$ can be deduced from an appropriate " $R=\boldsymbol{T}$ " theorem, since one proves that the maximal reduced quotient of $R^{\text {univ }}$ modulo an ideal of $R^{\text {loc }}$ is isomorphic to a finite $\mathbb{O}$-algebra $\boldsymbol{T}$. However, in dimension greater than 2, without a conjugate self-dual assumption, the current $R=\boldsymbol{T}$ theorems are contingent on conjectural properties of the cohomology of arithmetic quotients (see Part 2 of [Calegari and Geraghty 2012]).

We shall deduce from Theorem 1 the following corollaries:

Corollary 2. For any $\bar{\rho}$ satisfying the conditions of Theorem 1, Boston's strengthening of the unramified Fontaine-Mazur conjecture is equivalent to the unramified Fontaine-Mazur conjecture.

Corollary 3. Suppose that $\bar{\rho}: G_{F^{+}} \rightarrow \mathrm{GL}_{2}(k)$ satisfies the conditions of Theorem 1. Assume further that:

(1) $\bar{\rho}$ is totally odd.

(2) If $p=5$ and $\bar{\rho}$ has projective image $\mathrm{PGL}_{2}\left(\mathbb{F}_{5}\right)$, then $\left[F^{+}\left(\zeta_{5}\right): F^{+}\right]=4$.

Then Boston's conjecture holds: the representation $\rho^{\mathrm{unr}}: G_{F^{+}} \rightarrow \mathrm{GL}_{2}\left(R^{\mathrm{unr}}\right)$ has finite image.

When $n=2, p>2, F=\mathbb{Q}$, and $\bar{\rho}$ is totally odd and unramified at $p, R^{\mathrm{unr}}$ can be identified with the ring of Hecke operators acting on a (not necessarily torsion-free) coherent cohomology group (see [Calegari and Geraghty 2012]).

Let $\varphi_{n}$ be the group scheme over $\mathbb{Z}$ that is the semidirect product

$$
\left(\mathrm{GL}_{n} \times \mathrm{GL}_{1}\right) \rtimes\{1, J\}=\varphi_{n}^{0} \rtimes\{1, \jmath\},
$$

where $J$ acts on $\mathrm{GL}_{n} \times \mathrm{GL}_{1}$ by $J(g, \mu) J^{-1}=\left(\mu^{t} g^{-1}, \mu\right)$. Let $v: \mathscr{G}_{n} \rightarrow \mathrm{GL}_{1}$ be the character that sends $(g, \mu)$ to $\mu$ and $J$ to -1 . Let $F$ be a CM field with maximal totally real subfield $F^{+}$, and let

$$
\bar{r}: G_{F^{+}} \rightarrow \varphi_{n}(k)
$$

be a continuous homomorphism with $\bar{r}^{-1}\left(\varphi_{n}^{0}(k)\right)=G_{F}$. If $(A, \mathfrak{m})$ is a complete local 0 -algebra with residue field $k$, then a deformation $r$ of $\bar{r}$ to $A$ unramified outside a finite set of primes $S$ consists of an equivalence class of homomorphisms

$$
r: G_{F^{+}} \rightarrow \mathscr{G}_{n}(A)
$$

such that the composite of $r$ with the projection $\varphi_{n}(A) \rightarrow \varphi_{n}(A / \mathfrak{m})=\varphi_{n}(k)$ is $\bar{r}$, and such that the extension of fields $F(\operatorname{ker}(r))$ over $F(\operatorname{ker}(\bar{r}))$ is unramified away from places above primes in $S$. We say two lifts are equivalent if they are conjugate by an element of $\mathrm{GL}_{n}(A)$ that reduces to the identity modulo $\mathfrak{m}$. If $\bar{r}$ is Schur (see Definition 2.1.6 of [Clozel et al. 2008]), then this deformation problem is 
representable. By abuse of notation, we will again denote the universal deformation ring of $\bar{r}$ by $R^{\text {univ }}$ if $S$ contains all the primes above $p$, and by $R^{\mathrm{unr}}$ if $S$ contains no primes above $p$. This shouldn't cause any confusion, as we shall be very explicit regarding which deformation problem we refer to. As with the $\mathrm{GL}_{n}$-valued theory, for each $v \mid p$ in $F^{+}$, there is a universal framed deformation ring $R_{v}^{\square}$ which represents the lifts of $\bar{r} \mid D_{v}$, and a choice of lift in the equivalence class of the universal deformation of $\bar{r}$ makes $R^{\text {univ }}$ an algebra over

$$
R^{\mathrm{loc}}=\widehat{\bigotimes}_{v \mid p} R_{v}^{\mathrm{loc}}
$$

We shall deduce Theorem 1 from the following result.

Theorem 4. Let $F$ be a $C M$ field with maximal totally real subfield $F^{+}$. Let $S$ denote a finite set of places of $F^{+}$not containing any $v \mid p$, and let $\bar{r}: G_{F^{+}} \rightarrow \varphi_{n}(k)$ be a continuous homomorphism with $\bar{r}^{-1}\left(G_{n}^{0}(k)\right)=G_{F}$ and such that $v \circ \bar{r}\left(c_{v}\right)=-1$ for each choice of complex conjugation $c_{v}$. Assume that $p \geq 2(n+1)$, that the image of $\bar{r} \mid G_{F\left(\zeta_{p}\right)}$ is adequate, and that $\zeta_{p} \notin F$. Let $R^{\mathrm{unr}}$ be the universal deformation ring of $\bar{r}$ unramified outside $S$, and let $R^{\text {univ }}$ be the universal deformation ring of $\bar{r}$ unramified outside $S$ and all primes $v \mid p$. Then $R^{\mathrm{unr}}$ is a finite $\mathrm{O}$-algebra, and $R^{\mathrm{univ}}$ is a finite $R^{\text {loc }}$-algebra.

It turns out that the proof of this theorem is almost an immediate consequence of the finiteness results of [Thorne 2012] for ordinary deformation rings. The only required subtlety is to understand the relationship between the local ordinary deformation ring $R_{\Lambda_{K}}^{\Delta \text {,ar }}$ constructed in [Geraghty 2010] and the unramified local deformation ring $R^{\mathrm{un}}$.

\section{Some local deformation rings}

Recall $k$ is a finite field of characteristic $p$, and $O=W(k)$. Let $K$ be a finite extension of $\mathbb{Q}_{p}$ and let $G_{K}=\operatorname{Gal}(\bar{K} / K)$. Fix a continuous unramified representation

$$
\bar{\rho}: G_{K} \rightarrow \mathrm{GL}_{n}(k)
$$

and let $R^{\square}$ be its universal framed deformation ring. Let $R^{\text {un }}$ be the quotient of $R^{\square}$ corresponding to unramified lifts.

Lemma 5. The ring $R^{\mathrm{un}}$ is isomorphic to a power series ring over 0 in $n^{2}$ variables. In particular, it is reduced and its $\overline{\mathbb{Q}}_{p}$-points are Zariski dense in $\operatorname{Spec}\left(R^{\mathrm{un}}\right)$.

Proof. Fixing a choice of lift $g \in \mathrm{GL}_{n}(\mathcal{O})$ of $\bar{\rho}$ (Frob), it is easy to see that the lift to O【\{ $\left\{x_{i j}\right\}_{1 \leq i, j \leq n} \rrbracket$ given by Frob $\mapsto g\left(I+\left(x_{i j}\right)\right)$ is the universal framed deformation. 
Let $I_{K}^{\mathrm{ab}}$ be the inertia subgroup of the abelianization of $G_{K}$, and let $I_{K}^{\mathrm{ab}}(p)$ be its maximal pro- $p$ quotient. Let $\Lambda_{K}=\mathcal{O} \llbracket\left(I_{K}^{\mathrm{ab}}(p)\right)^{n} \rrbracket$ and let $\psi=\left(\psi_{1}, \ldots, \psi_{n}\right)$ be the universal $n$-tuple of characters $\psi_{i}: I_{K} \rightarrow \Lambda_{K}^{\times}$. Set $R_{\Lambda_{K}}^{\square}=R \widehat{\otimes}^{\square} \Lambda_{K}$.

We briefly recall the construction of the universal ordinary deformation ring $R_{\Lambda_{K}}^{\triangle}$ by Geraghty (see $\$ 3$ of [ibid.]). Let $\mathscr{F}$ be the flag variety over $\mathcal{O}$ whose $S$-points, for any $\mathbb{C}$-scheme $S$, is the set of increasing filtrations $0=F_{0} \subset F_{1} \subset \cdots \subset F_{n}=\mathbb{O}_{S}^{n}$ of $\mathbb{O}_{S}^{n}$ by locally free submodules with $\operatorname{rank}\left(F_{i}\right)=i$ for each $i=1, \ldots, n$. Lemma 3.1.2 of [ibid.] shows that the subfunctor of

$$
R_{\Lambda_{K}}^{\square} \otimes_{\mathcal{O}} \mathscr{F}
$$

corresponding to pairs $\left(\rho,\left\{F_{i}\right\}\right)$ such that

- $\left\{F_{i}\right\}$ is stabilized by $\rho$, and

- the action of $I_{K}$ on $F_{i} / F_{i-1}$ is given by the pushforward of $\psi_{i}$, is represented by a closed subscheme $\mathscr{G}$. He then defines $R_{\Lambda_{K}}^{\triangle}$ as the image of

$$
R_{\Lambda_{K}}^{\square} \rightarrow \mathrm{O}_{\varphi}(\mathscr{G}[1 / p]) .
$$

Since scheme-theoretic image commutes with flat base change, $R_{\Lambda_{K}}^{\triangle}[1 / p]$ is the scheme-theoretic image of

$$
\mathscr{G}[1 / p] \rightarrow \operatorname{Spec}\left(R_{\Lambda_{K}}^{\square}[1 / p]\right) .
$$

Since this map is proper, $\mathscr{G}[1 / p]$ surjects onto $\operatorname{Spec}\left(R_{\Lambda_{K}}^{\triangle}[1 / p]\right)$. Because $\mathscr{G}$ is of finite type over $R_{\Lambda_{K}}^{\triangle}$, we deduce that any $\overline{\mathbb{Q}}_{p}$-point of $\operatorname{Spec}\left(R_{\Lambda_{K}}^{\triangle}[1 / p]\right)$ lifts to a $\overline{\mathbb{Q}}_{p}$-point of $\varphi[1 / p]$. This proves the following.

Lemma 6. Let $x \in \operatorname{Spec}\left(R_{\Lambda_{K}}^{\square}\right)\left(\overline{\mathbb{Q}}_{p}\right)$, and let $\left(\rho_{x}, \psi_{x}\right)$ denote the pushforward via $x$ of the universal framed deformation and $n$-tuple of characters of $I_{K}$. Then $x$ factors through $R_{\Lambda_{K}}^{\triangle}[1 / p]$ if and only if there is a full flag $0=F_{0} \subset F_{1} \subset \cdots \subset F_{n}=\overline{\mathbb{Q}}_{p}^{n}$ stabilized by $\rho_{x}$ such that the action of $I_{K}$ on $F_{i} / F_{i-1}$ is given by $\psi_{i, x}$ for each $i=1, \ldots, n$.

If $\bar{\rho}$ is the trivial representation, then Geraghty defines a further quotient $R_{\Lambda_{K}}^{\triangle, a r}$ of $R_{\Lambda_{K}}^{\triangle}$ as follows. Let $Q_{1}, \ldots, Q_{m}$ be the minimal primes of $\Lambda_{K}$. For each $j=1, \ldots, m$, let $\mathscr{G}_{j}=\mathscr{G}_{\Lambda_{K}} \Lambda_{K} / Q_{j}$. Let $W_{j} \subset \operatorname{Spec}\left(\Lambda_{K} / Q_{j}\right)$ be the closed subscheme defined by $\psi_{r}=\epsilon_{p} \psi_{s}$ for some $1 \leq r<s \leq n$, and let $U_{j}$ be the complement of $W_{j}$. Geraghty shows (see $\S 3.4$ of [ibid.]) that there is a unique irreducible component $\varphi_{j}^{a r}$ of $\varphi_{j}$ lying above $U_{j}$. We then set $\varphi^{a r}=\bigcup_{1 \leq j \leq m} \varphi_{j}^{a r}$ and define $R_{\Lambda_{K}}^{\triangle, a r}$ to be the image of

$$
R_{\Lambda_{K}}^{\Delta} \rightarrow \operatorname{O} \operatorname{gar}\left(\mathscr{G}^{a r}[1 / p]\right) .
$$

The construction of $R_{\Lambda_{K}}^{\triangle, a r}$ together with Lemma 6 yields the following. 
Lemma 7. Assume that $\bar{\rho}$ is trivial. Let $x \in \operatorname{Spec}\left(R_{\Lambda_{K}}^{\square}\right)\left(\overline{\mathbb{Q}}_{p}\right)$, and let $\left(\rho_{x}, \psi_{x}\right)$ denote the pushforward via $x$ of the universal framed deformation and $n$-tuple of characters of $I_{K}$. Assume that there is a full flag $0=F_{0} \subset F_{1} \subset \cdots \subset F_{n}=\overline{\mathbb{Q}}_{p}^{n}$ stabilized by $\rho_{x}$ such that the action of $I_{K}$ on $F_{i} / F_{i-1}$ is given by $\psi_{i, x}$ for each $i=1, \ldots, n$. If $\psi_{i, x} \neq \epsilon_{p} \psi_{j, x}$ for any $i<j$, then $x$ factors through $R_{\Lambda_{K}}^{\triangle, a r}$.

Remark 8. If $\left[K: \mathbb{Q}_{p}\right]>\frac{1}{2} n(n-1)+1$ and $\bar{\rho}$ is trivial (which, for our applications, we could assume), then Thorne proves that $R_{\Lambda_{K}}^{\Delta, a r}=R_{\Lambda_{K}}^{\triangle}$ (see Corollary 3.12 of [Thorne 2014]).

There is a natural map $\Lambda_{K} \rightarrow R^{\text {un }}$ given by modding out by the augmentation ideal $\mathfrak{a}$ of $\Lambda_{K}$. We thus have a natural surjection

$$
R_{\Lambda_{K}}^{\square} \rightarrow R^{\mathrm{un}}
$$

Proposition 9. The surjection $R_{\Lambda_{K}}^{\square} \rightarrow R^{\mathrm{un}}$ factors through $R_{\Lambda_{K}}^{\Delta}$. If $\bar{\rho}$ is trivial, then it further factors through $R_{\Lambda_{K}}^{\triangle, a r}$.

Proof. The image of an unramified representation is the topological closure of the image of Frobenius. Since any element of $\mathrm{GL}_{n}\left(\overline{\mathbb{Q}}_{p}\right)$ is conjugate to an upper triangular matrix, that the image of any unramified representation into $\mathrm{GL}_{n}\left(\overline{\mathbb{Q}}_{p}\right)$ fixes a full flag for which the action of inertia on the corresponding quotients is trivial. It follows that the projection from $R_{\Lambda_{K}}^{\square}$ to any $\overline{\mathbb{Q}}_{p}$-point of $R^{\text {un }}$ factors through $R_{\Lambda_{K}}^{\Delta}$ by Lemma 6 and, if $\bar{\rho}$ is trivial, through $R_{\Lambda_{K}}^{\Delta, a r}$, by Lemma 7. The result then follows from the fact that $R^{\text {un }}$ is reduced and its $\overline{\mathbb{Q}}_{p}$-points are Zariski dense, by Lemma 5.

\section{Proof of Theorem 4}

We first prove the statement concerning $R^{\text {unr }}$ over 0 . Take a representation $\bar{r}$ as in Theorem 4. For each $v \mid p$ in $F^{+}$, let $F_{v}^{+}$be the completion of $F^{+}$at $v$ and let $\Lambda_{v}=\Lambda_{F_{v}^{+}}$with $\Lambda_{F_{v}^{+}}$as in Section 1. Let $\Lambda=\widehat{\bigotimes}_{v \mid p, 0} \Lambda_{v}$.

We note that, using Lemma 1.2.2 of [Barnet-Lamb et al. 2014], we are free to make any base change disjoint from the fixed field of $\operatorname{ker}(\bar{r})$. After a base change, we may assume that $\bar{r}$ is everywhere unramified, and that $\bar{r} \mid D_{v}$ is trivial for all $v \mid p$ as well as any finite set of auxiliary primes. In particular, after a suitable base change, we may restrict ourselves to considering deformation rings which are unipotent at some finite set of auxiliary primes $v \in S$ (which corresponds to the local deformation condition $R_{\tilde{v}}^{1}$ of [Thorne 2012, §8]). By Proposition 3.3 .1 of [Barnet-Lamb et al. 2014], we may assume, after a further base change, that $\bar{r}$ lifts to a minimal crystalline ordinary modular representation (this is where we use the assumption that $p \geq 2(n+1)$ ). From Corollary 8.7 of [Thorne 2012], we deduce that the corresponding ordinary deformation ring $R_{\mathscr{S}}$ is finite over $\Lambda$. If we can 
show that $R^{\text {unr }}$ is a quotient of $R_{\mathscr{S}} \otimes \Lambda / \mathfrak{a}$, where $\mathfrak{a}$ is the augmentation ideal of $\Lambda$, then the result follows immediately by Nakayama, since $\Lambda / \mathfrak{a}=0$. By definition, the local condition at $v \mid p$ for $R_{\varphi}$ is determined by the ordinary deformation ring $R_{\Lambda_{v}}^{\triangle, a r}$. By Proposition 9, the ring $R^{\mathrm{un}}$ is a quotient of $R_{\Lambda_{v}}^{\triangle, a r} / \mathfrak{a}$. Hence $R^{\mathrm{unr}}$ is a quotient of $R_{\mathscr{S}} \otimes \Lambda / \mathfrak{a}$ and we are done.

The finiteness of $R^{\text {univ }}$ over $R^{\text {loc }}$ then follows from the finiteness of $R^{\text {un }}$ over $O$ and Nakayama. Indeed, let $R^{\text {split }}=R^{\text {univ }} \otimes_{R^{\text {loc }}} k$ and let $r^{\text {split }}$ be the specialization of the universal deformation to $R^{\text {split }}$. Then $r^{\text {split }}\left|D_{v} \cong \bar{r}\right| D_{v}$ for any $v \mid p$ in $F^{+}$, so the quotient $R^{\text {univ }} \rightarrow R^{\text {split }}$ factors through $R^{\text {un }} \otimes_{\mathbb{0}} k$.

\section{Some corollaries}

3.1. Proof of Theorem 1. Let $\bar{\rho}$ satisfy the statement of Theorem 1. Consider $\operatorname{ad}^{0}(\bar{\rho})$ restricted to a suitable quadratic CM extension $F / F^{+}$. Since $p \nmid n$, the representation $\operatorname{ad}^{0}(\bar{\rho})$ is a direct summand of $\bar{\rho}^{c} \otimes \bar{\rho}^{*}=\bar{\rho} \otimes \bar{\rho}^{*}$ and is conjugate self-dual. The assumption of irreducibility together with the inequality $p>2 n^{2}-1$ imply that $\operatorname{ad}^{0}(\bar{\rho})$ is adequate by Theorem A.9 of [Thorne 2012]. If $n$ is even, then $\operatorname{ad}^{0}(\bar{\rho})$ has odd dimension and so is automatically totally odd. If $n$ is odd, then $\operatorname{ad}^{0}(\bar{\rho})$ is orthogonal (the conjugate self-duality is realized by the trace pairing, which is symmetric) and exactly self-dual (up to trivial twist) and so has trivial multiplier, which means that it is also totally odd. Both uses of totally odd refer to the properties of the multiplier character rather than the determinant of complex conjugation, and are the exact sign conditions required for automorphy lifting theorems for unitary groups (that is, totally odd means $U$-odd rather than GL-odd in the notation of [Calegari 2010]; see also §2.1 of [Barnet-Lamb et al. 2014]). Hence $\operatorname{ad}^{0}(\bar{\rho}) \mid G_{F}$ extends to a homomorphism (see Lemma 2.1.1 of [Clozel et al. 2008])

$$
\bar{r}: G_{F^{+}} \rightarrow \varphi_{n^{2}-1}(k),
$$

which we fix, satisfying the conditions of Theorem 4. On the other hand, any deformation of $\bar{\rho}$ gives rise to a deformation of $\bar{r}$ in the natural way. By Yoneda's lemma, there is a corresponding morphism $R^{\mathrm{unr}}(\bar{r}) \rightarrow R^{\mathrm{unr}}(\bar{\rho})$. It suffices to prove this is finite. By Nakayama's lemma, this reduces to showing that the only deformations $\rho$ of $\bar{\rho}$ to $k$-algebras such that $\operatorname{ad}^{0}(\rho)\left|G_{F} \cong \operatorname{ad}^{0}(\bar{\rho})\right| G_{F}$ are finite. The kernel of such a deformation must be contained in the maximal abelian pro- $p$ extension of $F(\operatorname{ker}(\bar{\rho}))$ unramified outside $S$, which is finite by class field theory. As in the final paragraph of the proof of Theorem 4, the finiteness of $R^{\mathrm{unr}}$ implies the finiteness of $R^{\text {split }}$ and hence that $R^{\text {univ }}$ is a finite $R^{\text {loc }}$-algebra.

If $n=2$ and $\bar{\rho}$ is totally odd, we may work directly with $\bar{\rho}$. We first use Corollary 1.7 of [Taylor 2002] to conclude that $\bar{\rho}$ is potentially modular and Theorem A of [Barnet-Lamb et al. 2013] to assume it is potentially ordinarily modular. Then, 
restricting $\bar{\rho}$ to a suitable $\mathrm{CM}$ field $F$, the proof is exactly as in the proof of Theorem 4 (without the appeal to Proposition 3.3.1 of [Barnet-Lamb et al. 2014]).

3.2. Proof of Corollary 2. This follows immediately from Theorem 1 and the following proposition.

Proposition 10. Let $F$ be a number field and let $\bar{\rho}: G_{F} \rightarrow \mathrm{GL}_{n}(k)$ be continuous and absolutely irreducible. Then

$$
\rho^{\mathrm{unr}}: G_{F} \rightarrow \mathrm{GL}_{n}\left(R^{\mathrm{unr}}\right)
$$

has finite image if and only if the following two properties hold:

(1) $R^{\mathrm{unr}}$ is finite over $\mathbb{0}$;

(2) for any minimal prime $\mathfrak{p}$ of $R^{\mathrm{unr}}[1 / p]$, the induced representation

$$
G_{F} \rightarrow \mathrm{GL}_{n}\left(R^{\mathrm{unr}}[1 / p] / \mathfrak{p}\right)
$$

has finite image.

Proof. If $\rho^{\mathrm{unr}}$ has finite image, then (2) is clearly satisfied, and (1) follows from Théorème 2 of [Carayol 1994], which shows that $R^{\text {unr }}$ is generated over $O$ by traces.

Now assume (1) and (2), and let $E$ be the fraction field of $\mathcal{O}$. Since $R^{\mathrm{unr}}$ is a finite 0 -algebra, the map $R^{\text {unr }} \rightarrow R^{\text {unr }}[1 / p]$ has finite kernel. Hence it suffices to prove that the map

$$
\rho: G_{F} \rightarrow \mathrm{GL}_{n}\left(R^{\mathrm{unr}}[1 / p]\right)
$$

has finite image, assuming (2). Since $R^{\mathrm{unr}}$ is finite over $\mathbb{O}$, the ring $R^{\mathrm{unr}}[1 / p]$ is a semilocal ring which is a direct sum of Artinian $E$-algebras $A$ with residue field $H$ for some finite $[H: E]<\infty$. In particular, the representation $\rho$ breaks up into a finite direct sum of representations to such groups $\mathrm{GL}_{n}(A)$. If $A=H$, then assumption (2) implies that the image of such a representation is finite. If $A \neq H$, then $A$ admits a surjective map to $H[\epsilon] / \epsilon^{2}$. In particular, there exists an unramified deformation

$$
\rho: G_{F} \rightarrow \mathrm{GL}_{n}\left(H[\epsilon] / \epsilon^{2}\right) .
$$

By assumption (2) again, the corresponding residual representation with image in $\mathrm{GL}_{n}(H)$ is finite, and is given by some representation $V$ on which $G_{F}$ acts through a finite group. Moreover, $\rho$ is then given by some nontrivial extension

$$
0 \rightarrow V \rightarrow W \rightarrow V \rightarrow 0 .
$$

Consider the restriction of this representation to a finite extension $L / F$ such that $G_{L}$ acts trivially on $V$. Then the action of $G_{L}$ on $W$ factors through an unramified $\mathbb{Z}_{p}$-extension, which must be trivial by class field theory. It follows that the action of $G_{L}$ on $W$ is trivial, and hence that the extension $W$ is trivial, a contradiction. 
3.3. Proof of Corollary 3. By Theorem 0.2 of [Pilloni and Stroh 2013] (see also [Kassaei 2013]), one knows the unramified Fontaine-Mazur conjecture for $\bar{\rho}$ under the given hypothesis, hence the result follows from Corollary 2.

\section{Acknowledgements}

We would like to thank Matthew Emerton, Toby Gee, and Vytautas Paškūnas for useful conversations. We would like to thank the organizers of the 2014 Bellairs workshop in number theory, where some of the ideas in this note were first discussed. We would also like to thank the referees for helpful comments on a previous version of this note.

\section{References}

[Barnet-Lamb et al. 2013] T. Barnet-Lamb, T. Gee, and D. Geraghty, "Congruences between Hilbert modular forms: constructing ordinary lifts, II”, Math. Res. Lett. 20:1 (2013), 67-72. MR 3126722 Zbl 06255950

[Barnet-Lamb et al. 2014] T. Barnet-Lamb, T. Gee, D. Geraghty, and R. Taylor, "Potential automorphy and change of weight", Ann. of Math. (2) 179:2 (2014), 501-609. MR 3152941 Zbl 06284344

[Boston 1999] N. Boston, "Some cases of the Fontaine-Mazur conjecture, II", J. Number Theory 75:2 (1999), 161-169. MR 2000b:11124 Zbl 0928.11050

[Calegari 2010] F. Calegari, "Even Galois representations", lecture notes, Institut Henri Poincaré, 2010, http://www.math.northwestern.edu/ fcale/papers/FontaineTalk-Adjusted.pdf.

[Calegari and Geraghty 2012] F. Calegari and D. Geraghty, "Modularity lifting beyond the TaylorWiles method", preprint, 2012, http://arxiv.org/abs/1207.4224. arXiv 1207.4224

[Carayol 1994] H. Carayol, "Formes modulaires et représentations galoisiennes à valeurs dans un anneau local complet", pp. 213-237 in p-adic monodromy and the Birch and Swinnerton-Dyer conjecture (Boston, MA, 1991), edited by B. Mazur and G. Stevens, Contemp. Math. 165, Amer. Math. Soc., Providence, RI, 1994. MR 95i:11059 Zbl 0812.11036

[Clozel et al. 2008] L. Clozel, M. Harris, and R. Taylor, "Automorphy for some $l$-adic lifts of automorphic mod $l$ Galois representations”, Publ. Math. Inst. Hautes Études Sci. 108 (2008), 1-181. MR 2010j:11082 Zbl 1169.11020

[Fontaine and Mazur 1995] J.-M. Fontaine and B. Mazur, "Geometric Galois representations", pp. 41-78 in Elliptic curves, modular forms, \& Fermat's last theorem (Hong Kong, 1993), edited by J. Coates and S.-T. Yau, Ser. Number Theory 1, Int. Press, Cambridge, MA, 1995. MR 96h:11049 Zbl 0839.14011

[Geraghty 2010] D. Geraghty, "Modularity lifting theorems for ordinary Galois representations", preprint, 2010, https://www2.bc.edu/david-geraghty/files/oml.pdf.

[Kassaei 2013] P. L. Kassaei, "Modularity lifting in parallel weight one", J. Amer. Math. Soc. 26:1 (2013), 199-225. MR 2983010 Zbl 1296.11052

[Mazur 1989] B. Mazur, "Deforming Galois representations", pp. 385-437 in Galois groups over $\mathbf{Q}$ (Berkeley, CA, 1987), edited by Y. Ihara et al., Math. Sci. Res. Inst. Publ. 16, Springer, New York, 1989. MR 90k:11057 Zbl 0714.11076

[Mazur 1997] B. Mazur, "An introduction to the deformation theory of Galois representations", pp. 243-311 in Modular forms and Fermat's last theorem (Boston, MA, 1995), edited by G. Cornell et al., Springer, New York, 1997. MR 1638481 Zbl 0901.11015 
[Pilloni and Stroh 2013] V. Pilloni and B. Stroh, "Surconvergence, ramification et modularité", preprint, 2013, http://perso.ens-lyon.fr/vincent.pilloni/Artinfinal.pdf.

[Taylor 2002] R. Taylor, "Remarks on a conjecture of Fontaine and Mazur", J. Inst. Math. Jussieu 1:1 (2002), 125-143. MR 2004c:11082 Zbl 1047.11051

[Thorne 2012] J. Thorne, "On the automorphy of $l$-adic Galois representations with small residual image”, J. Inst. Math. Jussieu 11:4 (2012), 855-920. Appendix by R. Guralnick, F. Herzig, R. Taylor and J. Thorne. MR 2979825 Zbl 1269.11054

[Thorne 2014] J. Thorne, "Automorphy lifting for residually reducible l-adic Galois representations", J. Amer. Math. Soc. (online publication June 2014).

Communicated by Brian Conrad

Received 2014-06-01 Revised 2014-09-22 Accepted 2014-11-12

pballen@math.northwestern.edu Mathematics Department, Northwestern University, 2033 Sheridan Road, Evanston, IL 60208, United States

fcale@math.northwestern.edu Department of Mathematics, Northwestern University, 2033 Sheridan Road, Evanston, IL 60208, United States 


\section{Algebra \& Number Theory}

msp.org/ant

\section{EDITORS}

MANAGING EDITOR

Bjorn Poonen

Massachusetts Institute of Technology

Cambridge, USA

\author{
EDITORIAL BOARD CHAIR \\ David Eisenbud \\ University of California \\ Berkeley, USA
}

BOARD OF EDITORS

Georgia Benkart

Dave Benson

Richard E. Borcherds

John H. Coates

J-L. Colliot-Thélène

Brian D. Conrad

Hélène Esnault

Hubert Flenner

Edward Frenkel

Andrew Granville

Joseph Gubeladze

Roger Heath-Brown

Craig Huneke

János Kollár

Yuri Manin

Barry Mazur

Philippe Michel

Susan Montgomery
University of Wisconsin, Madison, USA

University of Aberdeen, Scotland

University of California, Berkeley, USA

University of Cambridge, UK

CNRS, Université Paris-Sud, France

University of Michigan, USA

Freie Universität Berlin, Germany

Ruhr-Universität, Germany

University of California, Berkeley, USA

Université de Montréal, Canada

San Francisco State University, USA

Oxford University, UK

University of Virginia, USA

Princeton University, USA

Northwestern University, USA

Harvard University, USA

École Polytechnique Fédérale de Lausanne

University of Southern California, USA
Shigefumi Mori

Raman Parimala

Jonathan Pila

Anand Pillay

Victor Reiner

Peter Sarnak

Joseph H. Silverman

Michael Singer

Vasudevan Srinivas

J. Toby Stafford

Bernd Sturmfels

Richard Taylor

Ravi Vakil

Michel van den Bergh

Marie-France Vignéras

Kei-Ichi Watanabe

Efim Zelmanov

Shou-Wu Zhang
RIMS, Kyoto University, Japan

Emory University, USA

University of Oxford, UK

University of Notre Dame, USA

University of Minnesota, USA

Princeton University, USA

Brown University, USA

North Carolina State University, USA

Tata Inst. of Fund. Research, India

University of Michigan, USA

University of California, Berkeley, USA

Harvard University, USA

Stanford University, USA

Hasselt University, Belgium

Université Paris VII, France

Nihon University, Japan

University of California, San Diego, USA

Princeton University, USA

\section{PRODUCTION}

production@msp.org

Silvio Levy, Scientific Editor

See inside back cover or msp.org/ant for submission instructions.

The subscription price for 2014 is US \$225/year for the electronic version, and $\$ 400 /$ year ( $\$ 55$, if shipping outside the US) for print and electronic. Subscriptions, requests for back issues and changes of subscribers address should be sent to MSP.

Algebra \& Number Theory (ISSN 1944-7833 electronic, 1937-0652 printed) at Mathematical Sciences Publishers, 798 Evans Hall \#3840, c/o University of California, Berkeley, CA 94720-3840 is published continuously online. Periodical rate postage paid at Berkeley, CA 94704, and additional mailing offices.

ANT peer review and production are managed by EditFLOW ${ }^{\circledR}$ from MSP.

\section{PUBLISHED BY}

mathematical sciences publishers

nonprofit scientific publishing

http://msp.org/

(C) 2014 Mathematical Sciences Publishers 


\section{Algebra \& Number Theory}

Volume $8 \quad$ No. $9 \quad 2014$

Zeros of $L$-functions outside the critical strip

ANDREW R. BOOKER and FRANK THORNE

Tropical independence I: Shapes of divisors and a proof of the Gieseker-Petri theorem DAVID JeNSEN and SAM PAYNE

New equidistribution estimates of Zhang type

D. H. J. POLYMATH

2043

2067

Relations between Dieudonné displays and crystalline Dieudonné theory

2201

EIKE LAU

Finiteness of unramified deformation rings

2263

Patrick B. Allen and Frank Calegari

2273

On direct images of pluricanonical bundles

Minnea Popa and Christian SchNell 